\title{
Corrosion-passivation processes in a cellular automata based simulation study
}

\author{
Janusz Stafiej · Dung di Caprio · Eukasz Bartosik
}

Published online: 20 April 2013

(C) The Author(s) 2013. This article is published with open access at Springerlink.com

\begin{abstract}
A short survey of cellular automata based models for corrosion and passivation phenomena is given. Results of simulations based on the model of spatially separated anodic and cathodic reactions are presented for a cavity development from a point-like damage of a protective layer and from an initially flat unprotected surface. We show some new peculiar examples of the symmetry breaking in the cavity development. The results for the initially flat surface show roughening of the surface at the beginning of the corrosion process. After that, pit merging causes a resmoothing of the surface. An oscillatory behavior of the surface roughness is observed caused by a peninsula formation with subsequent island detachment. These results are obtained in $2 \mathrm{D}$ because of computational limitations. We plan simulations in $3 \mathrm{D}$ and point out the problems we encounter in their realization.
\end{abstract}

Keywords Corrosion $\cdot$ Stochastic cellular automata $\cdot$ Surface roughness $\cdot$ Chunk effect

\section{Introduction}

Cellular automata (CA) are used in science to model the behavior of many real systems. The range of problems that can be approached in this way is rather wide and spanning over apparently unrelated domains such as public transport systems [3], plant ecosystems [1], forest fire spreading [2], cell cultures [4], bacterial colonies

J. Stafiej $(\varangle) \cdot$ Ł. Bartosik

Department of Complex Systems and Chemical Processing of Information, Institute of Physical Chemistry, Polish Academy of Sciences, Kasprzaka 44/52, 01-224 Warsaw, Poland e-mail: jstafiej@ichf.edu.pl

D. di Caprio

Laboratory Electrochemistry, Chemistry of Interfaces and Modelization for the Energy (LECIME), ENSCP, CNRS, 4, Pl. Jussieu, 75005 Paris, France 
[5], plant morphogenesis [6]. Since their invention [7], CA play an outstanding role in computer science [8]. For example, CA are implied in Turing machine and computability [9], neural networks [10], pattern recognition [11], to name some from the list of applications.

CA are particularly useful to study phenomena that appear in a wider class of systems of various chemical nature. Then we can assume a common physicochemical origin for them. Corrosion and passivation are a good example [13, 14]. Both occur on several different metals and alloys like aluminum, zinc, iron, steel-to name the most economically important. They differ in their physicochemical properties. They are relatively durable in usual conditions although the free enthalpy of their reaction with water, oxygen and other chemical species makes them thermodynamically unstable and subject to corrosion. They owe their durability to passivation that is formation of the layer of corrosion products on the surface. The passive layer can reduce the corrosion rate by many orders of magnitude separating the bare metal surface from the environment. The way the passive layer plays its protective role and mechanisms of its breakdown have been an active subject of an intense research on both theoretical and experimental sides for a long time in view of the economical importance of the corrosion prevention $[15,16]$. Cellular automata models for this phenomenon [13] illustrate the known paradox: the higher reactivity of the surface, the faster passive layer formation and the better surface protection. Thus in an aggressive environment characterized by higher bare corrosion rates the effective corrosion rates are lower than in a mild environment where passive layer formation is slower.

The passive layer breakdown even by a small local damage may lead to pitting corrosion. This is an insidious form of corrosion as the development of the corrosion pit may escape attention, pierce through a layer of material and destroy the function of the object, as in the case of containers storing dangerous waste especially in a liquid or gaseous form. This form of corrosion is also studied in recent CA models [17]. In these studies, metal surface is covered by an insulating layer put in contact with an aggressive solution. The paint layer cuts electrical connections and imposes open circuit conditions on the cavity development. In the first stage of this process, small depassivated spots formed in depassivation events heal soon by repassivation when diffusion is efficient enough to mix and neutralize anodic and cathodic solutions. Beyond a critical cavity size, diffusion fails to keep cavity interior neutral. Anodic spots develop at acidified pits. Then the surface crosses over to a faster corrosion development accelerated by an autocatalytic feedback in the anodic dissolution inside pits. The feedback loop consists in that the dissolution products acidify the environment. The acidification reduces repassivation and increases depassivation. The inner pit surface remains exposed to a further anodic dissolution, further increasing acidification. In contrast, passive layers are more stable in basic medium at cathodic region and protected against acidity.

In this paper we reconsider this model. It is used mainly to describe development of a cavity initiated by a punctual damage of the insulating layer covering the surface. In a recent paper we considered this model also for a planar surface [18] concentrating on a single pit development and its morphology. Here we sketch the general outline of the model (Sect. 2) as it is already presented elsewhere, summarizing the main results of our earlier published work for damage induced pit development (Sect. 3). 


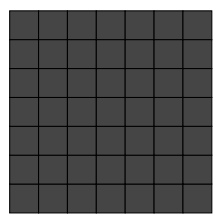

Metal

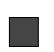

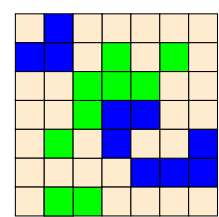

Solution

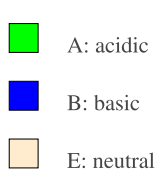

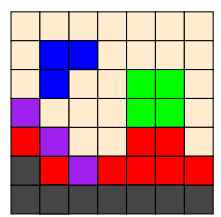

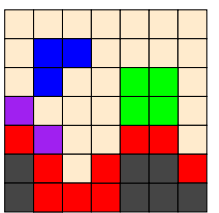

$\mathrm{R}$ : reactive sites

P: passive sites

Fig. 1 Cellular automata model for corrosion

We present further examples of a peculiar pit development starting from the punctual damage. We also show some results on surface evolution starting from the planar surface exposed to the environment. In Sect. 4 we give suggestions for a future work.

\section{Cellular automata model for corrosion}

\subsection{Lattice representation of the corroding system}

As sketched in Fig. 1, we use a square lattice. The lattice site can be in six states or, in other words, occupied by six species. These are: bulk metal $M$, two surface metal sites $R, P$ on the metal side, and three solution sites $E, A, B$ respectively neutral, acidic and basic. If according to some rules a surface metal site is removed, its $M$ neighbors become reactive sites. We use either von Neumann 4 connectivity or, most often, Moore 8-connectivity that yields a larger acidity-basicity scale compared to von Neumann 4-connectivity [12]. The Moore connectivity has also another advantage discussed later.

\subsection{Transformation rules for the system evolution}

In our CA model we consider several stochastic processes. Spatially separated electrochemical (SSE) reactions present one of such processes. The anodic part of this reaction affects only $R$ sites. At a given time step, we select at random from all $R$ sites those sites that attempt to react. The reaction pathway depends on neighborhood of the $R$ site. The neighborhood is characterized by $N_{\mathrm{nn}}^{\mathrm{exc}}$ which is the algebraic excess of $A$ over $B$ sites as illustrated in Fig. 2. This quantity is roughly related to local $\mathrm{pH}$ at the site and it is positive for low and negative for high $\mathrm{pH}$. If $N_{\mathrm{nn}}^{\mathrm{exc}} \geq 0$ :

$$
R \longrightarrow A
$$

else:

$$
R+B(\mathrm{nn}) \longrightarrow P+E(\mathrm{nn})
$$


Fig. 2 Examples of acidic and basic site environments
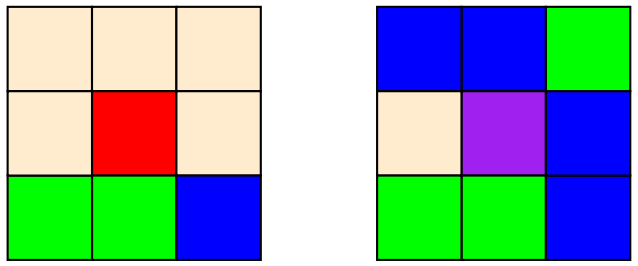

The (nn) indicates the nearest neighbor site. We assume that both kinds of surface site $S$, either $S=R$ or $S=P$, can participate in the cathodic reaction with an a priori equal probability. However, the $R$ site for the anodic reaction and its cathodic partner $S$ must be joined by a path of $R, P$ or $M$ nearest neighbors. In other words, they must belong to a single piece of metal. This rule reflects the fact that the metal is an electron conductor while the solution is an ionic conductor. The path of bulk and surface metal sites mediates the electron transfer between the two sites. Electrons cannot pass through solution sites.

The cathodic reaction can neutralize a neighboring acidic site:

$$
S+A(\mathrm{nn}) \longrightarrow S+E(\mathrm{nn})
$$

or basify a neutral site:

$$
S+E(\mathrm{nn}) \longrightarrow S+B(\mathrm{nn}) .
$$

Both reactions tend towards a local acidification of the solution. Note that the coupled anodic and cathodic reactions obey a conservation law related to the charge conservation. The difference in the number of $A$ and $B$ species is conserved since always a pair of new $A$ and $B$ sites is created. If there is an existing $A$ or $B$ site involved, one of them can be annihilated and almost instantaneously recreated elsewhere. We are tempted to call it "teleportation," although in contrast to the use of this term for paranormal phenomena, it is the electric signal that transmits information between the two sites rather than instantaneous matter displacement. "Teleportation" with neutralization amounts to a simultaneous removal of a pair of sites $A$ and $B$. Of course, "teleportation" works only between sites adjacent to a single connected set of metal sites. For each $R$ site the SSE reactions have an a priori probability, $p_{\text {sse }}$, to be realized. However, the a posteriori probability of this reaction depends on the blocking of its cathodic part by the presence of $B$ sites.

In consistence with the random walk rules discussed later, we apply a simple exclusion rule. If all the solution nearest neighbors of the surface site are of $B$ type, this site cannot mediate the cathodic process. We repeat the selection from among the other $S$ sites until a free site is found or until the list of connected surface sites is exhausted. In the latter case the anodic reaction is canceled as we fail to find the cathodic counterpart.

If the electrochemical reaction proceeds at the same site then the $A$ and $B$ compensate and the reactions amount to for basic medium

$$
R \longrightarrow P
$$


We associate the following basicity/acidity dependent probabilities with this event:

$$
P_{R P}= \begin{cases}0 & \text { if } N_{\mathrm{nn}}^{\operatorname{exc}}>0 \\ p_{\mathrm{cor} 1} & \text { if } N_{\mathrm{nn}}^{\operatorname{exc}}=0 \\ 1 & \text { if } N_{\mathrm{nn}}^{\operatorname{exc}}<0\end{cases}
$$

where passivation is promoted in basic medium, and for acidic medium we assume

$$
R \longrightarrow E
$$

The $M$ nearest neighbors of the destroyed active site become $R$ sites.

The anodic process and SSE reactions are not possible on the totally passivated surface. The passivity breakdowns bring the bare metal surface to contact with the solution. We represent such events by:

$$
\begin{aligned}
P & \longrightarrow E, \\
M(\mathrm{nn}) & \longrightarrow R .
\end{aligned}
$$

The probability of this process is also related to the local acidity/basicity:

$$
P_{P E}= \begin{cases}0 & \text { if } N_{\mathrm{nn}}^{\text {exc }}<0 \\ p_{\mathrm{oxi}} & \text { if } N_{\mathrm{nn}}^{\mathrm{exc}}=0, \\ 0.25 N_{\mathrm{nn}}^{\mathrm{exc}} & \text { if } N_{\mathrm{nn}}^{\text {exc }}>0\end{cases}
$$

We assume that in contact with a basic environment a reactive site gets immediately passivated. With the choice of our parameters we should like to mimic the fact that the surface is hard to depassivate: $p_{\text {oxi }} \sim 0$. The species $B$ and $A$ execute a random walk over $E$ sites according to the following rules. At each time step, a nearest neighbor of these sites is selected at random. If the nearest neighbor is an $E$ site, the walkers execute a swap according to:

$$
A B_{1}+E_{2} \longrightarrow E_{1}+A B_{2}
$$

where $A B=A$ or $A B=B$. The subscripts " 1 " and " 2 " denote the source and the target site for the random step. If the walker and the neighbor are a pair of $A$ and $B$, they both neutralize each other:

$$
A+B \longrightarrow E_{1}+E_{2}
$$

no matter what site is the source and the target for the step. In other cases, the species $A$ and $B$ stay where they are. The nature of the neighbor site remains also unchanged:

$$
A B_{1}+X_{2} \longrightarrow A B_{1}+X_{2}
$$

Note that random walk and annihilation are consistent with the conservation rule of the SSE reactions. To regulate the diffusion rate with respect to corrosion rate in a simple way, we introduce an integer number $N_{\text {diff. }}$ For each time step related 
Fig. 3 Initial conditions for the simulations starting from a local damage (a) and a planar interface with periodic boundary conditions (b)

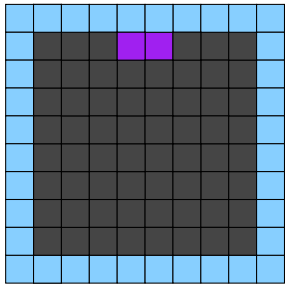

a)

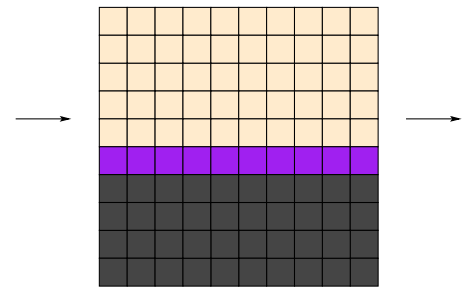

b) protective layer

to corrosion, we perform $N_{\text {diff }}$ steps of random walk assuming that the diffusion processes are faster than the corrosion ones. For simplicity we assume that both $A$ and $B$ execute random walk at the same rate.

The above schemes and the values that we use for the set of probabilities are only qualitative and conceived on what is generally known about corrosion processes in some class of materials [19]. The generic features of the corrosion process do not depend, however, on the detailed form of our assumptions.

The results presented below have been obtained in a simulation box of size $1000 \times$ 1000 sites. For the cavity development study the initial configuration is a block of $998 \times 998 M$ sites bordered by a frame of inert wall sites imitating a non-conductive protective layer. Two $M$ sites at the positions $(500,999)$ and $(501,999)$ are then turned into $R$ sites imitating a local damage of the protective layer and putting the $M$ sites in contact with the environment. This initial configuration and an alternative one for the planar interface are shown in Fig. 3. Then the rules of transformation described above launch the front evolution.

\subsection{Connectivity algorithm for the SSE reactions}

The most difficult part of the algorithm is checking the connectivity required by the simultaneous realization of SSE reactions. It seems reasonable to think that the surface of a connected set is itself a connected set and to look for the path connecting two surface sites only at the surface. This is however not true for the von Neumann connectivity because the nearest neighbors of a site are disconnected as a set if you remove the site. In contrast, the nearest neighbors of a site form a connected set in Moore connectivity. In both Moore and von Neumann choices for "bulk" connectivity we define surface connectivity. Two surface sites are surface nearest neighbors when either they are von Neumann nearest neighbors of each other or they have a common $M$ site as a von Neumann nearest neighbor. It is easy to see that von Neumann connectivity, surface connectivity and Moore connectivity are equivalence relations. The surface connectivity allows us to find surfaces of von Neumann connected or Moore connected sets by applying whichever algorithm for finding surface connectivity equivalence classes in the set of all surface sites. It would be redundant and more time-consuming to apply von Neuman or Moore connectivity to all metal sites and check whether the $R$ and $S$ sites are in the same bulk connected subset. Surface connectivity class may contain several von Neumann classes of the surface sites and be a part of a larger Moore connectivity class. 
In the initial implementation we used an elegant iteration scheme based on C construction of a function calling itself, which from site to site labels all sites pertaining to a cluster. It led however to stack overflows and we switched to a less elegant but more stable explicit depth-first search method [20]. The simulations presented here were performed on a Quad Dual Xeon $2.5 \mathrm{GHz}$ PC. Single simulation run took up to 7 hours. The code is written in $\mathrm{C}$ and compiled with gcc version 4.1.2. The simulations of a similar but simpler problem avoiding connectivity checking in $3 \mathrm{D}$ for a similarly sized $1000 \times 1000 \times 500$ box take a week on CUDA GPU Tesla C2050. Our first attempts to implement the connectivity checking extended this time to 1 month and led to introduction of a different approach to circumvent the connectivity problem. We do not see however how to apply the circumvention to more complicated problem of electrochemical reaction and are looking for another solution that would make 3D simulations more practical.

\section{Results}

The results of the simulations starting from the initial condition as in Fig. 3a are already published and discussed [17, 18]. Here we show some additional examples illustrating the peculiar behavior of the cavity shape and the main features of these results.

In a large domain of explored parameters the development of the cavity follows the same scenario. Initially the development of the corrosion cavity is isotropic and uniform characterized by a low corrosion rate due to a small depassivation probability. Initially the cavity shape is roughly a semicircle. It is neutral, as for the small cavity size diffusion is able to bring about mutual neutralization of $B$ and $A$ sites. This breaks down as soon as the cavity size goes beyond a critical size defined by an average incubation radius $R_{I}$ after an average incubation time $t_{I}$. The zones of cathodic passivated sites appear. The solution gets basified in the vicinity of these zones. In the rest of the surface the reactive sites acidify the neighboring solution when dissolved in the anodic reaction. The zone separation leads to a speedup of corrosion by a simultaneous pit creation and pit merging process. We see a crossover from a slower to a higher corrosion rate. At this crossover the cavity shape is extremely unstable, depending on the random pattern of zones created by spontaneous symmetry breaking. An example of such a pattern formation and its further development is given in Fig. 4. In Fig. 4a we see the initial stage of the zone separation. The cavity resembles in shape a beetle with an abdomen, thorax and head. The abdomen and head are acidified and progress via anodic dissolution while the thorax in the middle is basified and preserves the rough structure of healed small pits resembling insect legs (Fig. 4b). Finally the head turns up and stops progressing due to basification (Fig. 4c). It makes us think about the lacy cover effect where the corroded cavity is covered by a layer of uncorroded material and the cavity development can make holes in the cover around the original orifice [23]. Another example (Fig. 5) shows how insidious localized corrosion can be proceeding inwards to the material and leaving the cavity orifice small. In both cases we see with bare eyes the relative smoothness of the anodic zones as compared to rough cathodic zones. This feature 

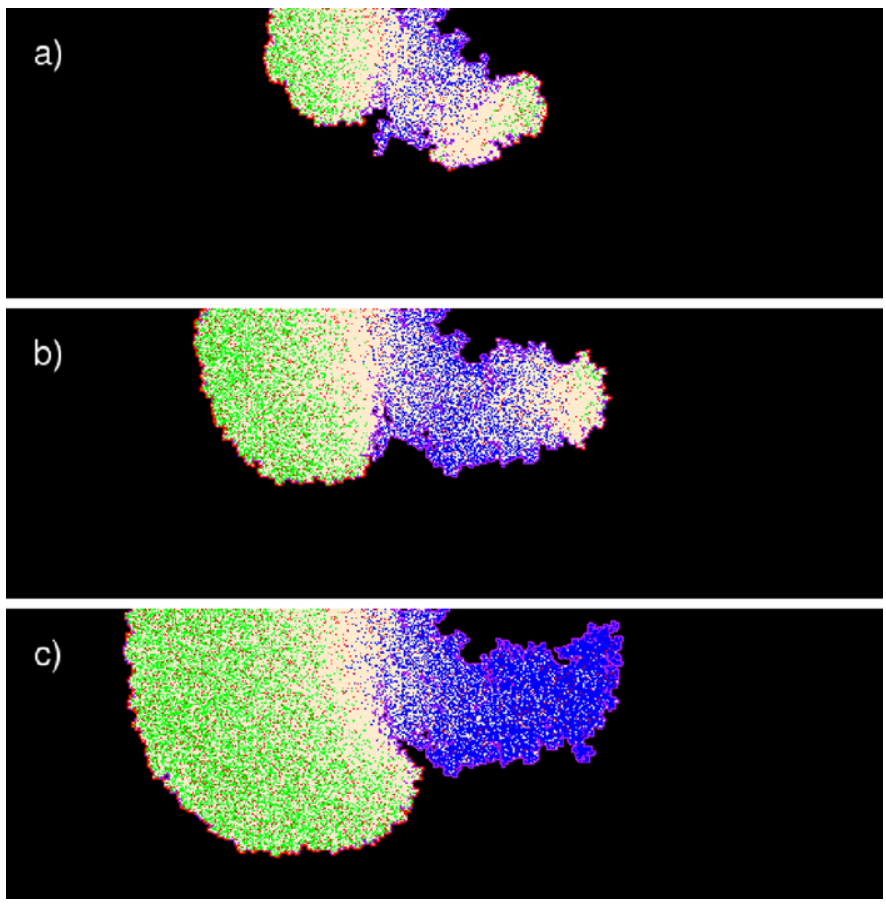

Fig. 4 Cavity development that may lead to a lacy cover formation. The time steps are 1020, 1080 and 1140 for $(\mathbf{a}),(\mathbf{b})$ and $(\mathbf{c})$, respectively, and $p_{\text {oxi }}=0.001, p_{\text {cor } 1}=0.0, p_{\text {cor } 2}=0.0, N_{\text {diff }}=100$

is observed experimentally. It appears at an early stage of zone separation as seen in Fig. 6 for a higher diffusion rate of $A$ and $B$. Here the rough structure of cathodic region is clearly seen to form from small anodic regions arising spontaneously and hiding in the pores against the neutralizing effect of the basic majority that preserves this structure. In contrast, anodic dissolution smooths out the pores dissolving material around them. This peculiar behavior of symmetry breaking and formation of rough cathodic and smooth anodic regions is experimentally found in experiments on corrosion of aluminum foil in quasi 2D experiments [24, 25].

When the cavity size is large compared to the incubation radius, the cavity shape assumes again a roughly circular form as the random symmetric breaking averages out at lengths much larger than the incubation radius. The solution may seem isotropic again. However, magnifying the image near the surface reveals that the solution is composed of basic and acidic regions that determine a higher rate constant of dissolution after the incubation.

Let us present some results on the evolution in time of an initially planar front. We first study the effect of the delocalization of the anodic and cathodic reactions in a geometry which is no longer confined. In Fig. 7, starting from the case where there are no delocalized reactions, we observe for increasing values of the probability $p_{\text {sse }}$ the evolution of the corrosion front. We see that both the corrosion rate and the roughness increase with increasing the delocalization probability of the anodic and cathodic reactions. The effect of the probability $p_{\text {sse }}$ is to increase the heterogeneity 

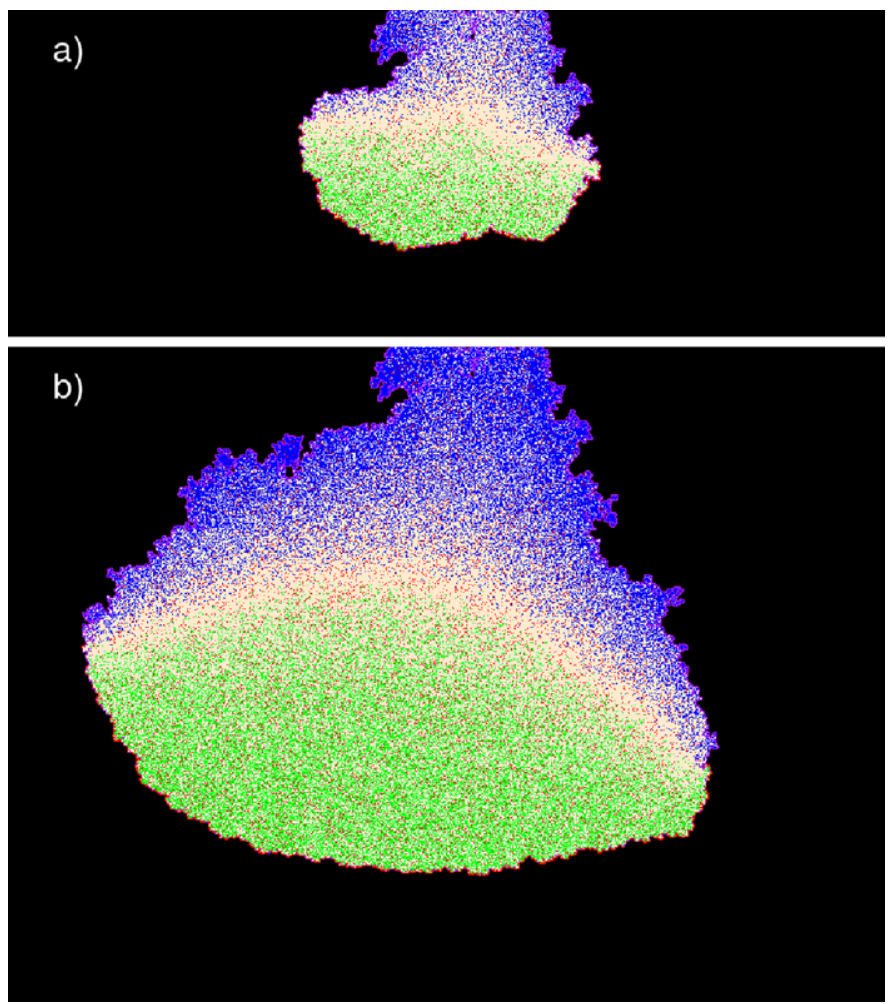

Fig. 5 Insidious cavity development inwards to the corroded material. The time steps are 1060 and 1380 for (a) and (b), respectively. Parameters identical to those of Fig. 4, only the initial random seed is modified

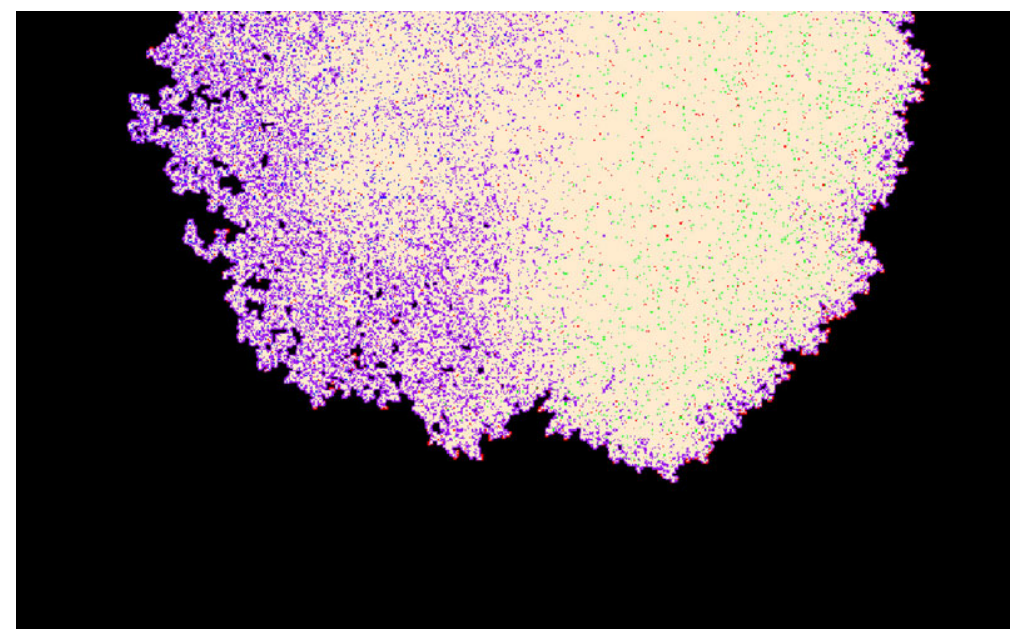

Fig. 6 Initial stage of zone separation for a higher diffusion rate $N_{\text {diff }}=10,000$ at the time step 1580 and remaining parameters identical to Fig. 4 

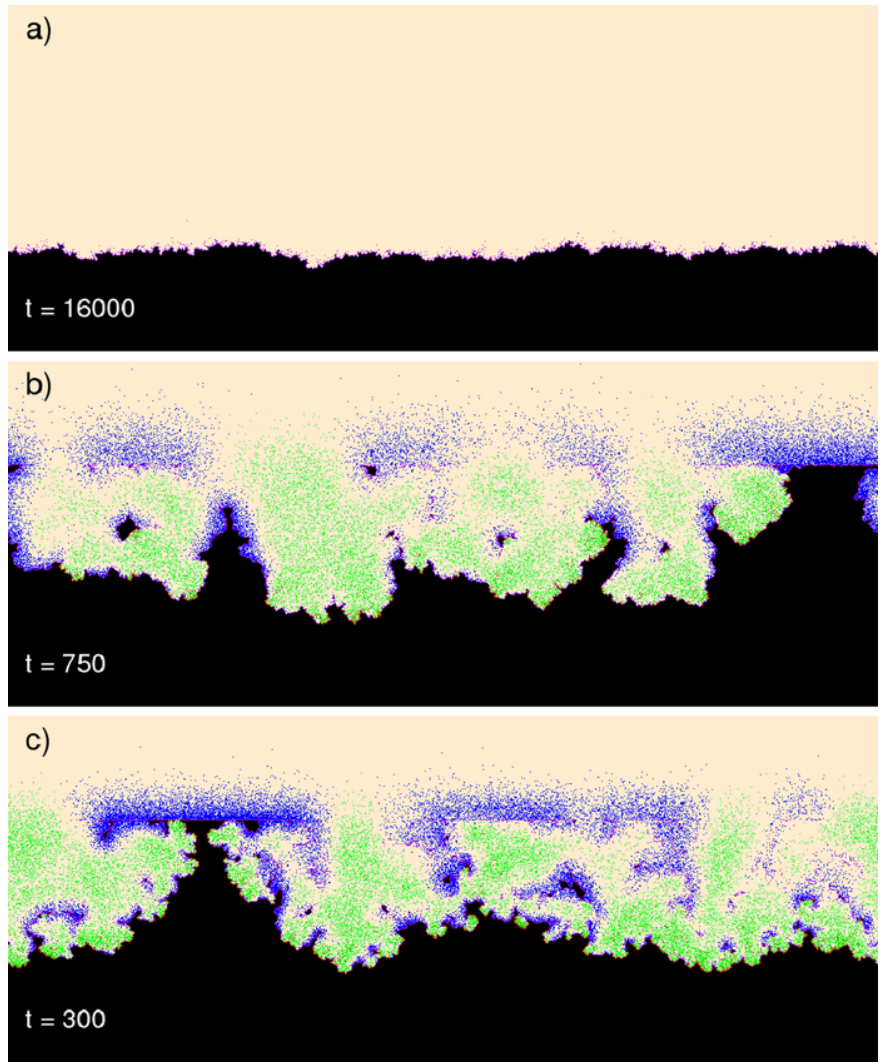

Fig. 7 Snapshots of the corroding surface for equivalent corrosion depths and different time steps as indicated. Simulation parameters are as follows: $p_{\text {oxi }}=0.005, p_{\text {cor } 1}=0.99, p_{\text {cor } 2}=0.002, N_{\text {diff }}=2$ and $p_{\text {sse }}=0.0,0.15,0.50$ respectively for $(\mathbf{a}),(\mathbf{b})$ and (c). The metal $M$ is at the bottom and the solution at the top. The color codes are shown in Fig. 1

of the corrosion front with, in particular, the appearance of acidic regions with higher corrosion rates resulting in a more pronounced roughness of the corrosion front.

In Fig. 8 we show snapshots at selected values of simulation time for an intermediate value of $p_{\text {sse }}$. We observe an evolution from the initial flat state to a surface covered by corrosion pits. The pits develop and merge $(t=1650)$. When merging is mostly done, a relatively smooth surface reappears. Then large irregularities $(t=3550)$ of the surface show up again. The irregularities take form of fancy shaped peninsulas. The snapshots of the corrosion front from $t=1400$ to $t=1650$ show how the peninsula gets insulated from the main front and becomes an island. After the island detachment, the front is smoother. The island then gets corroded $(t=1650$ and $t=1850$ ), attacked mostly in the acidic region adjacent to it. This peculiar behavior of the corrosion front, alternatively smooth and rough, is seen in Fig. 9, which gives the plot of the front roughness as measured by $\sigma$, the root of the mean square deviation of the front position from its average. 

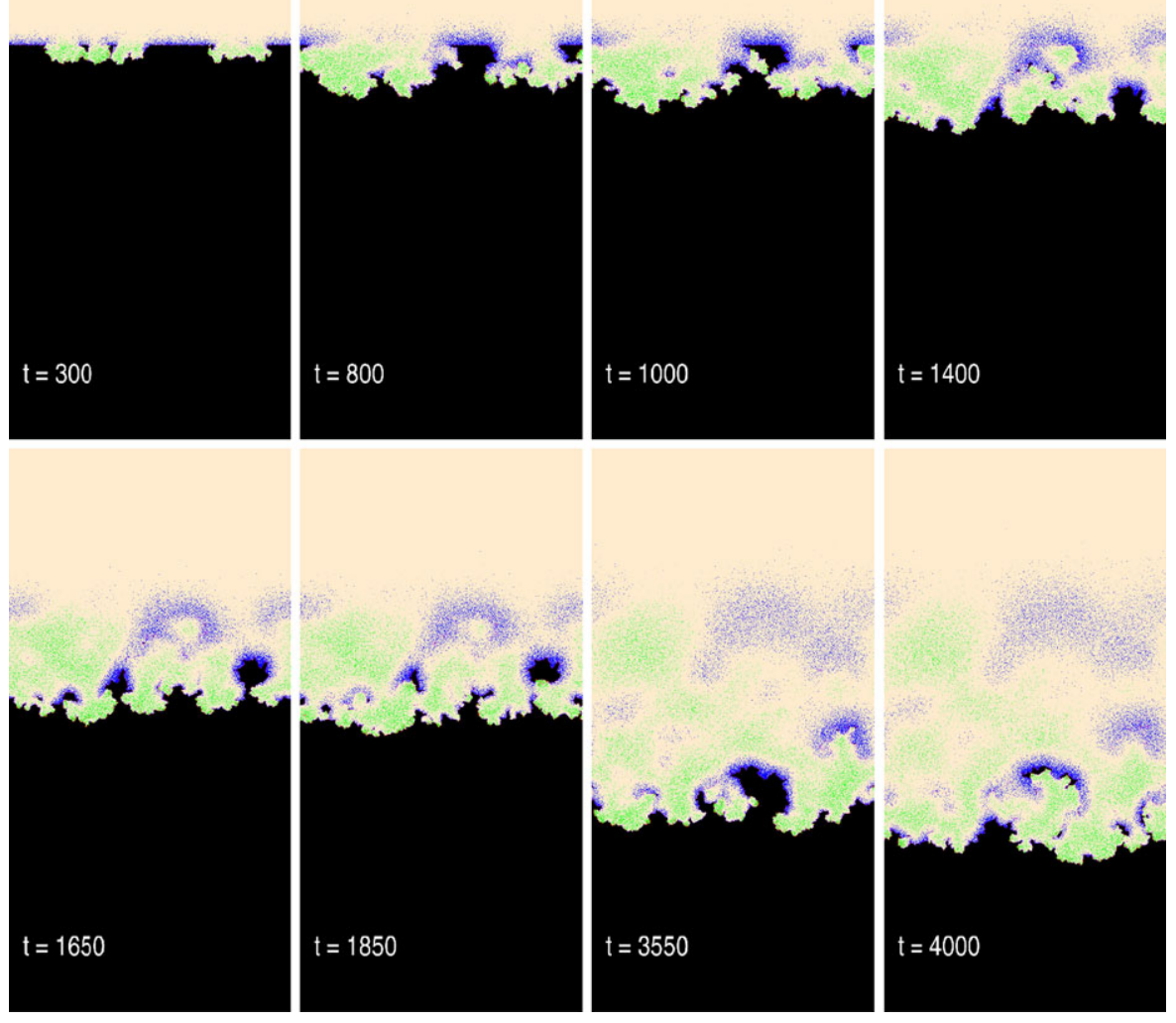

Fig. 8 Snapshots of the corroding surface at selected time steps. Simulation parameters are as in Fig. 7 with $p_{\text {sse }}=0.15$

The front roughness shows oscillatory behavior owing to alternative pit formation, pit merging and detachment of islands from corroded peninsulas. Island detachment is observed already for other models of corrosion and in the real world experiments. It gives an explanation for anomalous dissolution as the chunk effect [21]. The discussion of this effect, in the case of silver dissolution, dating back to 1958 corresponds well to our model [26]. A more recent account on the chunk effect can also be found [27].

Here we should explain why we illustrate our simulations with just a single run. It does not differ qualitatively from about a hundred of independent similar runs and illustrates a typical situation. However, averaging over these runs gives a featureless quasi-monotonous curve with no resemblance to the typical roughness profile. Clearly we cannot describe correctly this corrosion process by an average roughness profile. Averaging loses a vital part of information that we perceive visually even if we do not know how to describe it quantitatively. 
Fig. 9 Front roughness versus simulation type step

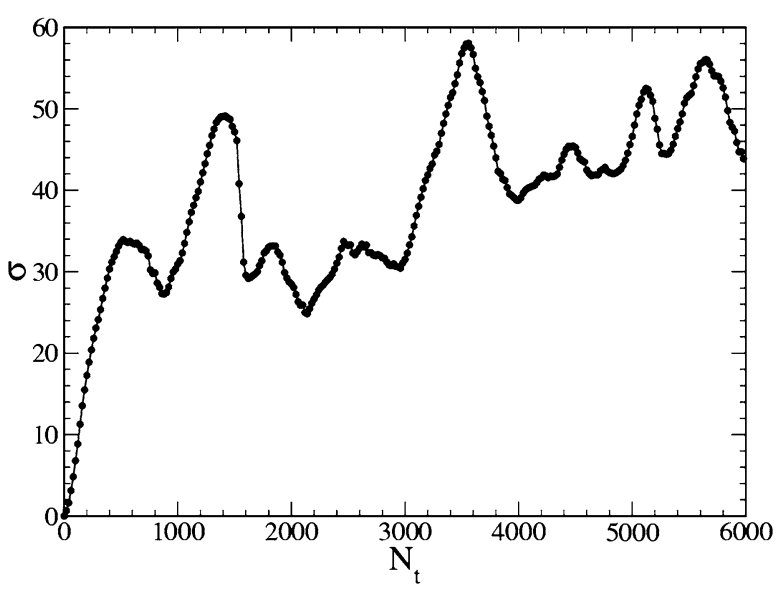

\section{Perspectives}

The CA modeling gives us a possibility to predict complicated morphologies and phenomena such as the peninsula formation and island detachment (chunk effect). At present, we develop models and methods to understand how the passivity breakdown in the passive layer may occur and how to influence the function and morphology of the passive layer. By fine-tuning parameters describing passive layer formation and the competing passive layer dissolution we may hope to elaborate etching protocols that yield desired morphologies of the treated surfaces. However, our simulations in 2D may only give us some first intuition and it is necessary to proceed to more realistic simulations in 3D. It is known that the neutralization reaction in $1 \mathrm{D}$ and $2 \mathrm{D}$ in homogeneous systems yields nontrivial pattern formation of zones of either $B$ or $A$ hardly observed in 3D [22]. One problem in passing to 3D is an efficient connectivitychecking algorithm. We can expect that limiting connectivity check to the surface can reduce the computational load for this problem.

Acknowledgements This work has been financed by the Polish Ministry of Science and Higher Education, the French Ministries of National Education and Research, and The French Ministry of Foreign Affairs within the framework of bilateral agreement POLONIUM 20106YH, and Polish Ministry's of Science and Higher Education Grant No. N N204 139038. One of us (L.B.) acknowledges the Ph.D. fellowship of the Foundation for Polish Science.

Open Access This article is distributed under the terms of the Creative Commons Attribution License which permits any use, distribution, and reproduction in any medium, provided the original author(s) and the source are credited.

\section{References}

1. Dunn AG, Majer JD (2007) Simulating weed propagation via hierarchical, patch-based cellular automata. In: Computational science. Lecture notes in computer science-ICCS 2007, vol 4487. Springer, Berlin, pp 762-769

2. Hernández Encinas A, Hernández Encinas L, Hoya White S, Martín del Rey A, Rodríguez Sánchez G (2007) Simulation of forest fire fronts using cellular automata. Adv Eng Softw 38:372-378 
3. Chowdhury D, Santen L, Schadschneider A (2000) Statistical physics of vehicular traffic and some related systems. Phys Rep 329:199-329

4. Lee Y, Kouvroukoglou S, McIntire L, Zygourakis K (1995) A cellular automaton model for the proliferation of migrating contact-inhibited cells. Biophys J 69:1284-1298

5. Wimpenny JWT, Colasanti R (2006) A unifying hypothesis for the structure of microbial biofilms based on cellular automaton models. FEMS Microbiol Ecol 22:1-16

6. Lazareva GG, Mironova VV, Omelyanchuk NA, Shvab IV, Vshivkov VA, Gorpinchenko DN, Nikolaev SV, Kolchanov NA (2008) Mathematical modeling of plant morphogenesis. Numer Anal Appl $1: 123-134$

7. Beyer WA, Sellers PH, Waterman MS (1985) Stanislaw M. Ulam's contributions to theoretical theory. Lett Math Phys 10:231-242

8. Wolfram S (2002) A new kind of science. Wolfram Media Inc, Champaign

9. Thatcher JW (1970) Self-describing Turing machines and self-reproducing cellular automata. In: Burks AW (ed) Essays on cellular automata. University of Illinois Press, Champaign, pp 103-131

10. Krundel LA (2008) Neural networks with cellular automata. PhD project, Loughborough University, UK. Presented on symposium on neural networks and cellular automata: from superconducting junctions to biology, Loughborough University, UK, February 4-5

11. Raghavan $R$ (1993) Cellular automata in pattern recognition. Inf Sci 70:145-177

12. Chopard B, Droz M (1998) Cellular automata modeling of physical systems. Cambridge University Press, Cambridge

13. di Caprio D, Stafiej J (2010) Simulations of passivation phenomena based on discrete lattice gas automata. Electrochim Acta 55:3884-3890

14. di Caprio D, Stafiej J (2011) The role of adsorption in passivation phenomena modelled by discrete lattice gas automata. Electrochim Acta 56:3963-3968

15. Landoldt D (2003) Traité des matériaux. Chimie de surfaces des métaux. Les Presses Universitaires et Polytechniques Romandes, Lausanne

16. Szklarska-Smialowska Z (2004) Pitting and crevice corrosion. NACE, Houston

17. Reis FDAA, Stafiej J, Badiali JP (2006) Scaling theory in a model of corrosion and passivation. J Phys Chem B 110:17554-17562

18. Bartosik $Ł$, di Caprio D, Stafiej J (2013) Cellular automata approach to corrosion and passivity phenomena. Pure Appl Chem 85:247-256

19. Frankel GS (1998) Pitting corrosion of metals; a summary of the critical factors. J Electrochem Soc 145:2186-2198

20. Cormen TH, Leiserson CE, Rivest RL, Stein C (2001) Introduction to algorithms, 2nd edn. MIT Press and McGraw-Hill, Cambridge, pp 540-549. Sect. 22.3: Depth-first search

21. Vautrin-Ul C, Mendy H, Taleb A, Chaussé A, Stafiej J, Badiali JP (2008) Numerical simulations of spatial heterogeneity formation in metal corrosion. Corros Sci 50:2149-2158

22. Argyrakis P, Kopelman R (1992) Diffusion controlled binary reactions in low dimensions: refined simulations. Phys Rev A 45:5814-5819

23. Laycock NJ, White SP (2001) Computer simulation of single pit propagation in stainless steel under potentiostatic control. J Electrochem Soc 148:B264-B275

24. Balazs L (1996) Corrosion front roughening in two-dimensional pitting of aluminium thin layers. Phys Rev E 54:1183-1189

25. Balazs L, Gouyet JF (1995) Two-dimensional pitting corrosion of aluminium thin layers. Physica A 217:319-338

26. Vermilyea DA (1958) The nature of anode slime. J Electrochem Soc 105:547-548

27. Guo HX, Lu BT, Luo JL (2006) Non-Faraday material loss in flowing corrosive solution. Electrochim Acta 51:5341-5348 